\title{
Computed tomography for diagnosing graves ophtalmopathy
}

\author{
Mihai Leonard C. Grecescu \\ ${ }^{1}$ Emergency University Hospital, Bucharest, Romania \\ 2"Carol Davila" University of Medicine and Pharmacy, Bucharest, Romania
}

\begin{abstract}
Graves ophtalmopathy (GO), thyroid eye disease (TED) and thyroid - associated orbitopathy (TAO) are terms referring to an autoimmune inflammatory process that affects the adipogenesis of orbital fat and periorbital tissue, associated with dysthyroidism. On imaging, such as computed tomography (CT) thyroid -associated orbitopathy (or thyroid -associated ophtalmopathy) is characterised by symmetrical and bilateral proptosis, secondary to extra-ocular muscle (EOM) enlargement and expansion of orbital fat. Imaging studies ca be useful in identifying patients risk to develop dysthyroid optic neuropathy and initiating early treatment of the condition, avoiding permanent visual loss.

The purpose of this study was to reveal the prevalence of thyroid ophtalmopathy imaging considerations in Graves disease patients. A total of 19 patients were evaluated on computed tomography scanning the diameters and density of extra-ocular muscles, muscular index, proptosis, width of the optic nerve, width of superior ophthalmic vein and lacrimal gland position.
\end{abstract}

Keywords: Graves ophtalmopathy, optic neuropathy, thyroid, computed tomography, extraocular muscles enlargement, proptosis

\section{INTRODUCTION}

Graves disease is one of the most common autoimmune condition, with an annual incidence in women of 1 in 1,000 [1]. Approximately $6 \%$ of patients with Graves ophtalmopathy develop optic neuropathy $(\mathrm{ON})$ [2]. Extra-thyroidal manifestations of Graves disease (Graves orbitopathy), occurring in $25-50 \%$ of patients with the disease $[3,4]$. The clinical connection between immunogenic hyperthyroidism and orbitopathy suggests that the antigen responsible for these diverse conditions may be shared by the thyroid gland and orbital tissues [5].

Physiopathologic theories about the ethology of GO suggested that inflammation of the extra-ocular muscles and connective tissue are caused by the presence of anti -TSH receptor antibodies and anti-insulin growth factor receptor 1 (IGF-1R) [6].

Diagnostic imaging methods such as CT indicate increased volume in the affected extra-ocular muscles (EOMs) and in the orbital fat, together or separately. Computed tomography (CT) is the most commonly used imaging technique for evaluating GO. CT is more sensitive than magnetic resonance imaging (MRI) in identifying enlarged extra-ocular muscles [7]. Findings on CT scanning may include muscle belly enlargement that often spares the tendinous insertions, an apparent increase in orbital fat volume and crowding of the optic nerve at the orbital apex [8]. CT can distinguish normal structures from abnormal structures of different tissue density based on their differing $\mathrm{X}$-ray absorption properties.

In Graves ophtalmopathy the muscles are infiltrated with inflammatory cells (lymphocytes, macrophages, plasma cells, eosinophils) and increased mucopolysaccharide deposition leads to fibrosis. The exactly mechanism is unknown, antibodies to thyroid-stimulating hormone (TSH) appear to cross-react with antigens in the orbit resulting in infiltration by activated $\mathrm{T}$ lymphocytes, with subsequent release of inflammatory mediators [9]. 
There are two distinct stages of thyroid orbitopathy. First is the inflammatory phase (16-34 months) characterised by interstitial oedema, lymphocytic infiltration and glycosaminoglycans deposition in enlarged extra-ocular muscles and retroorbital fat. The second phase (inactive disease) is characterised by fibrosis and fat infiltration of the orbital tissues [10-13].

Involvement of the extra-ocular muscles in decreasing order of frequency is: levator muscle, inferior rectus, medial rectus, superior rectus, lateral rectus, obliques. The involvement is bilateral in $76-90 \%$ and symmetric in 70\% [9].

Computed tomography (CT) scanning is an accurate imaging procedure for the diagnosis of thyroid ophtalmopathy.

Due to its widespread availability and fast image acquisition, the diagnosis is often first made on CT scan. Intravenous contrast can reveal the different densities of orbital fat and muscles for an adequate delineation of the orbital contents.

The most characteristic CT findings in thyroid orbitopathy include: exophthalmos (the distance between interzygomatic line and posterior sclera is 9.9+/-1.7 the distance between interzygomatic line to the anterior surface of the globe is normally $<23$ $\mathrm{mm}$; if the distance is $>23 \mathrm{~mm}$ it indicates exophtalmos), extra-ocular muscle enlargement and fatty attenuation, the "Coca-Cola bottle" sign (the anterior tendon is spared with the swelling largely confined to the muscle belly), increase of the retro-ocular orbital fat [9].

The reference normal size range for the extra-ocular muscles are: inferior rectus: $3.2-6.5 \mathrm{~mm}$, medial rectus: 3.3-6.1 mm, superior group: 3.2-6.1 $\mathrm{mm}$, lateral rectus: $1.7-4.8 \mathrm{~mm}$.

The size of the muscles correlates with the severity of disease and the risk of optic nerve compression [9].

Increase of width of medial rectus muscle seems to be most reliably connected with optic neuropathy development $[14,15]$.

Other findings include proptosis and anterior prolapse of the orbital septum due to enlargement of muscles and orbital fat compartment and intracranial fat prolapse [16].

There are some signs that reveal a high risk of developing optic neuropathy: severe apical crowding, enlargement and anterior displacement of the lacrimal gland, dilated superior ophthalmic vein, bowing of the medial lamina papyracea to accommodate the swollen-bulge of the medial rectus.

The most sensitive indicator for the presence of optic neuropathy is the apical crowding. The enlargement of the extra-ocular muscle bulges con- verged at the crowded orbital apex and induce compression of the optic nerve and causes visual decrease [16].

\section{PURPOSE}

The purpose of this retrospectively study was to reveal the role of the extra-ocular muscles enlargement in proptosis development.

\section{MATERIALS AND METHODS}

The study retrospectively evaluated 38 orbits of 19 patients (3 males and 16 females) ophtalmologically assessed with thyroid ophtalmopathy before CT examination.

Patients range in age from 35 to 67 years (mean 51 years).

All scannings were performed without intravenous contrast material.

The patients were required to maintain a gentle eye closure and forward gaze to prevent asymmetric extra-ocular muscle contraction [17].

Computed tomography examination was performed with a spiral CT scanner (Philips Brilliance 16), in axial $2 \mathrm{~mm}$ thick, pitch $=1 \mathrm{scans}$, parallel to the infraorbitomeatal line. After scanning were performed coronal and multi-planar reconstructions of the orbit.

All the parameters (diameters and density of extra-ocular muscles, proptosis, width of the optic nerve-sheath complex, the length of the interzygomatic line, muscular index, width of superior ophthalmic vein and lacrimal gland position) were calculated on direct axial and reconstructed coronal CT scans and interpreted as correct or incorrect reported to standard CT measurements for orbital structures [18-21].

Ranges for the densities of extra-ocular muscles were assessed in axial scans. Maximal diameters of extra-ocular muscles were measured perpendicularly to the orbital wall in the planes parallel in axial scans (medial rectus and lateral rectus) (Figure 1) and in coronal scans (inferior rectus, superior oblique and superior group - superior rectus and levator palpebrale superior muscle) (Figure 2).

The globe position was measured as the perpendicular distance between the interzygomatic line and the posterior limit (Figure 1).

The mean optic nerve-sheath was measured perpendicularly to its course in the axial CT sections in retrobulbar and middle segment of the optic nerve (Figure 1). 
The interzygomatic line was measured from the anterior edges at the zygomatic bones at the midglobe section (Figure 1).

The width of superior ophthalmic vein was measured in axial scans.

The enlargement and anterior displacement of the lacrimal gland was determined on axial images.

The horizontal muscular index was established as a percentage of orbital width (C) occupied by the medial rectus (A) and lateral rectus (B) muscles at a point halfway between posterior globe and the orbital apex (Figure 3).

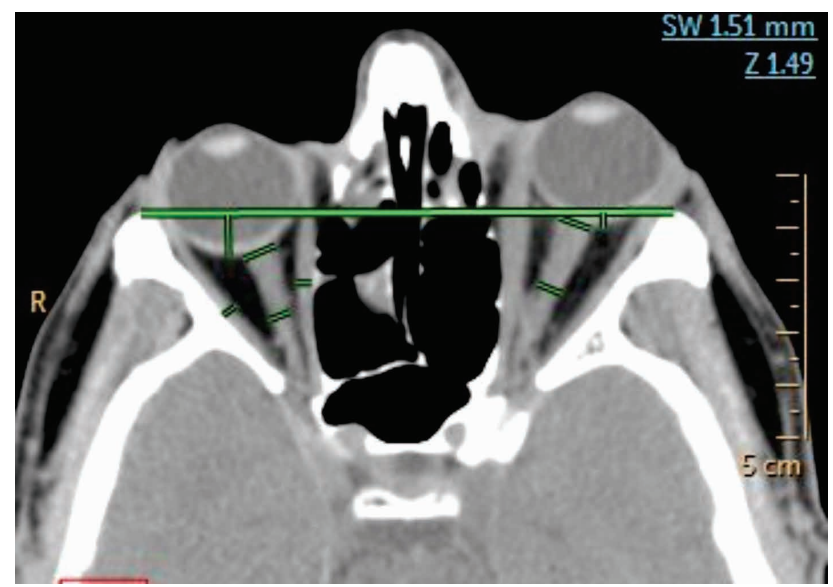

FIGURE 1. Axial CT scan - measurement for Interzygomatic line, globe position and optic nerve sheath measurement

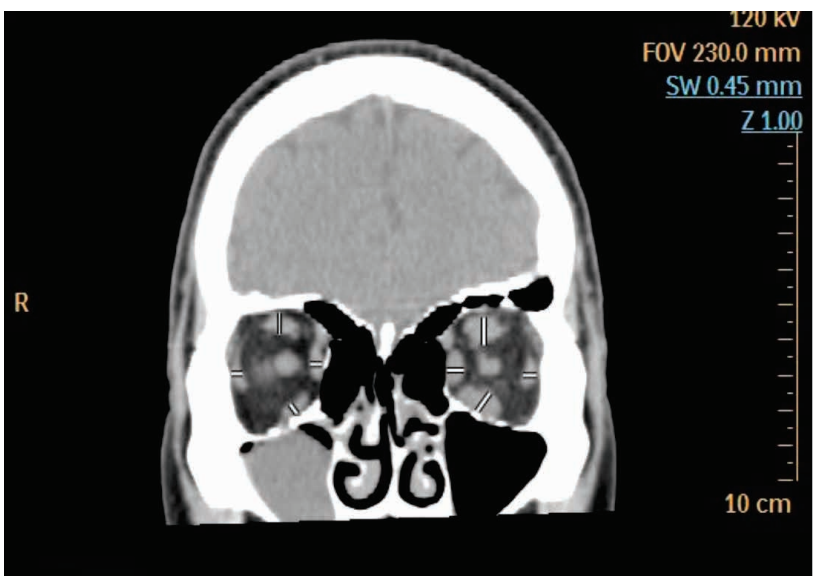

FIGURE 2. Coronal CT scan - measurement for extra-ocular muscle

The calculation formula was: $\{[(\mathrm{A}+\mathrm{B}) / \mathrm{C}] / 100\}$ (Figure 3). The vertical muscular index was expressed as a percentage of orbital heigh (F) occupied by the superior group muscles (superior rectus and levator muscle complex) (D) and inferior rectus muscle (E) at a point halfway between posterior globe and the orbital apex.

The calculation formula was similar with horizontal muscular index: $\{[(\mathrm{D}+\mathrm{E}) / \mathrm{F}] / 100\}$ (Figure 3).

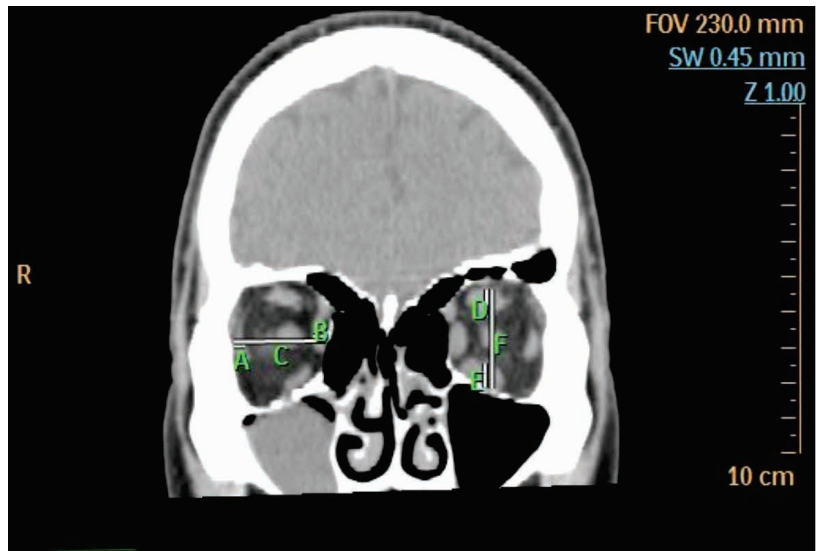

FIGURE 3. Coronal CT scan - measurement for muscular index

The values of this 2 indices reveal the significant impingement on the optic nerve space.

The values of the muscular index were used to compare right and left orbit data. Correlations of paired samples were calculated using the Pearson correlation coefficient.

\section{RESULTS}

Computed tomography imaging reveal that $77.7 \%$ (28/36 orbits) of the patients present proptosis, while $79 \%$ cases of diagnosed proptosis were bilateral.

Proptosis caused by expansion of orbital fat was observed mainly in patients under the age of 40 years.

Extra-ocular muscle diameter measured in this study revealed approximately the same values compared with references data (26/36 orbits, $72.2 \%$ with increased muscular index) [18-21].

Mean diameter ratio for muscular index were: medial rectus 1.29 , lateral rectus 1.32 , inferior rectus 1.37, superior group 1.31 and superior oblique 1.18 .

The most enlarged muscle were the inferior rectus $(59 \%)$ and medial rectus (52\%) and than superior group (37\%), lateral rectus (35\%) and superior oblique $(24 \%)$.

The highest correlation was revealed between proptosis and muscle enlargement in the cases of inferior rectus (75\%) and medial rectus (68\%).

$79 \%$ of muscle enlargement cases were bilateral and $21 \%$ unilateral.

Anterior displacement of the lacrimal gland was remarked in $46 \%$ cases.

There was no difference in mean axial superior ophthalmic vein diameter in any case.

There were no statistically significant differences between left and right orbit results. 
TABLE 1. Orbital measurement compared to normal value - according to (19)

\begin{tabular}{|c|c|c|c|}
\hline \multirow{2}{*}{ Muscles } & & $\begin{array}{c}\text { Range } \\
(\mathbf{m m})\end{array}$ & $\begin{array}{c}\text { Normal ranges } \\
(\mathbf{m m})\end{array}$ \\
\hline \multirow{3}{*}{} & Superior group & $3.2-6.5$ & $3.2-6.1$ \\
\cline { 2 - 4 } & Inferior rectus & $3.2-6.1$ & $3.2-6.5$ \\
\cline { 2 - 4 } & Medial rectus & $3.3-6.1$ & $3.3-5.0$ \\
\cline { 2 - 4 } & Lateral rectus & $1.7-4.8$ & $1.7-4.8$ \\
\cline { 2 - 4 } & Superior oblique & $1.8-4.3$ & - \\
\hline Globe position & & $0.7-6.7$ & $5.9-12.8$ \\
\hline Interzygomatic line & & $90.7-106.2$ & $91-108$ \\
\hline Muscular index(\%) & & $50.1-68.3$ & - \\
\hline Superior opthalmic vein & $1.4-2.3$ & $1.4-2.3$ & - \\
\hline \multirow{2}{*}{ Optic nerve } & Retrobulbar & $4.6-5.9$ & $3.2-5.6$ \\
\cline { 2 - 4 } & Middle segment & $3.5-5.2$ & \\
\hline
\end{tabular}

Assessment of all the parameters according to standard CT data for orbital structures are described in Table 1.

\section{DISCUSSION}

Computed tomography (CT) scanning is an accurate imaging procedure for the diagnosis of thyroid ophtalmopathy. Beside the visualisation of the enlarged extra-ocular muscles and the proptosis extent, CT scans provide an accurate image of the orbit when orbital decompression is required.

According to The European Group on Graves' Orbitopathy (EUGOGO) imaging of the orbits by CT or MRI scanning [21] is recommended when clinical or serological data are uncertain, in the case of suspected dysthyroid optic neuropathy or unilateral disease. CT scan can also exclude coexisting pathology and to indicate the degree of muscular involvement or to evaluate the result of orbital decompression surgery [22].

Computed tomography scanning is useful in detected the patients with increased risk of orbital complications like optic nerve involvement at the orbital apex. This CT scan highly sensitive in revealing of optic neuropathy with apical crowding (induced by the muscles and/ fat tissue) seems to be the most specific indicator of intraorbital pathology. The conclusions of this study confirm the results of other studies that the most frequently affected muscle in Graves orbitopathy is the inferior rectus, followed by superior and lateral rectus and after by superior oblique [10,21]. Three among 38 orbits $(7,9 \%)$ did not manifest proptosis, despite enlarged muscle diameter. In this lot of patients there was an exceeded muscular index in $50 \%$ of all the cases. This observation confirm the theory that an exceeded muscular index above $50 \%$ is a positive indicator of apical crowding.

Enlargement of the extra-ocular muscle diameter and the muscles density decrease at all muscle groups, implying that fat and inflammation in dysthyroid orbitopathy do not impair individual orbital elements, but the entire orbital compartment [22].

\section{CONCLUSIONS}

Computed tomography (CT) is generally the preferred imaging modality for the diagnosis of patients with Graves' orbitopathy because of its ability to visualise bone and soft tissues in the orbit. CT also aids the evaluation of the orbital walls, sinus and orbital elements in orbital decompression surgical intervention.

CT imaging can provide evaluation data in inflammatory disease activity. Changes observed with $\mathrm{CT}$ in sequential measurements of the extra-ocular muscles may be correlated with clinical activity (muscular involvement occurs early in Graves' orbitopathy).

Specification of parameters such as thickness and density in numerical values indicate $\mathrm{CT}$ as a proper monitoring method in thyroid orbitopathy.

\section{REFERENCES}

1. Pieroni Goncalves AC, Gebrim EMM, Monteiro MLR. Imaging studies for diagnosis Graves' orbitopathy and dysthyroid optic neuropathy. Clinics. 2012;67(11):1327-1334.

2. Bartley GB, Gorman C.A. Diagnostic criteria for Graves'ophtalmopathy. Am J Ophtalmol.1995;119(6):792-5.
3. Smith TJ. Pathogenesis of Graves' orbitopathy: a 2010 update. J Endocrinol Invest. 2010;33(6):414-21.

4. Bahn RS, Heufelder AE. Pathogenesis of Graves' ophtalmopathy. N Engl J Med.1993;329(20):1468-75.

5. Heufelder AE. Pathogenesis of ophtalmopathy in autoimmune thyroid disease. Rev Endocr Metab Disord. 2000 Jan;1(1-2): 87-95. 
6. Kazim M, Goldberg RA, Smith TJ. Insights into the pathogenesis of thyroid-associated orbitopathy: evolving rationale for therapy. Arch Ophthalmol. 2002 Mar;120(3):380-6.

7. Mourits MP, Koornneef $L$, Wiersinga WM, Prummel MF, Berghout $A$, van der Gaag R. Clinical criteria for the assessment of disease activity in Graves' ophthalmopathy: a novel approach. $\mathrm{Br} \mathrm{J}$ Ophthalmol. 1989 Aug;73(8):639-44.

8. Char DH. Thyroid Eye Disease. (3rd ed.). Boston: ButterworthHeinemann, 1997.

9. Rose JG Jr, Burkat CN, Boxrud CA. Diagnosis and management of thyroid orbitopathy. Otolaryngol Clin North Am. 2005 Oct; 38(5):1043-74

10. Scott IU, Siatkowski MR. Thyroid eye disease. Semin Ophthalmol. 1999 Jun;14(2):52-61.

11. Denniston A, Dodson P, Reuser T. Diagnosis and management of thyroid eye disease. Hosp Med. 2002 Mar;63(3):152-6.

12. Kazim M, Goldberg RA, Smith TJ. Insights into the pathogenesis of thyroid-associated orbitopathy: evolving rationale for therapy. Arch Ophthalmol. 2002 Mar;120(3):380-6.

13. Mourits MP, Koornneef L, Wiersinga WM, Prummel MF, Berghout A, van der Gaag R. Clinical criteria for the assessment of disease activity in Graves' ophthalmopathy: a novel approach. $\mathrm{Br} \mathrm{J}$ Ophthalmol. 1989 Aug;73(8):639-44.

14. Hallin ES, Feldon SE. Graves' ophthalmopathy: I. Simple CT estimates of extraocular muscle volume. Br J Ophthalmol. 1988 Sep;72(9):674-7.

\section{Conflict of interest: none declared}

Financial support: none declared
15. Hallin ES, Feldon SE. Graves' ophthalmopathy: II. Correlation of clinical signs with measures derived from computed tomography. $\mathrm{Br} \mathrm{J}$ Ophthalmol. 1988 Sep;72(9):678-82.

16. Giaconi JA, Kazim M, Rho T, Pfaff C. CT scan evidence of dysthyroid optic neuropathy. Ophthalmic Plast Reconstr Surg. 2002 May;18(3):177-82.

17. Chen YL, Chang TC, Huang KM, Tzeng SS, Kao SC. Relationship of eye movement to computed tomographic findings in patients with Graves' ophthalmopathy. Acta Ophthalmol (Copenh). 1994 Aug;72(4):472-7.

18. Nugent RA, Belkin RI, Neigel JM, Rootman J, Robertson WD, Spinelli J, Graeb DA. Graves orbitopathy: correlation of CT and clinical findings. Radiology. $1990 \mathrm{Dec} ; 177(3): 675-82$.

19. Ozgen A, Ariyurek M. Normative measurements of orbital structures using CT. AJR Am J Roentgenol. 1998 Apr;170(4):1093-6.

20. Lee JS, Lim DW, Lee SH, Oum BS, Kim HJ, Lee HJ. Normative measurements of Korean orbital structures revealed by computerized tomography. Acta Ophthalmol Scand. 2001 Apr;79(2):197-200.

21. Sheikh M, Abalkhail S, Doi SA, Al-Shoumer KA. Normal measurement of orbital structures: implications for the assessment of Graves' ophthalmopathy. Australas Radiol. 2007 Jun;51(3):253-6.

22. Fidor-Mikita E. Computed tomography imaging of orbits in thyroid orbitopathy. J Pre Clin Clin Res. 2008;2(1):59-63. 